\title{
Philosophiques
}

Thomas Nagel, Égalité et partialité (traduit par $\mathrm{Cl}$.

Beauvillard), Paris, Presses Universitaires de France (coll. « Philosophie morale »), 1994, vi-199 pages.

\section{Paul Dumouchel}

Volume 23, numéro 1, printemps 1996

Critères esthétiques et métamorphoses du beau

URI : https://id.erudit.org/iderudit/027382ar

DOI : https://doi.org/10.7202/027382ar

Aller au sommaire du numéro

Éditeur(s)

Société de philosophie du Québec

ISSN

0316-2923 (imprimé)

1492-1391 (numérique)

Découvrir la revue

Citer ce compte rendu

Dumouchel, P. (1996). Compte rendu de [Thomas Nagel, Égalité et partialité (traduit par Cl. Beauvillard), Paris, Presses Universitaires de France (coll. « Philosophie morale »), 1994, vi-199 pages.] Philosophiques, 23(1), 183-185. https://doi.org/10.7202/027382ar d'utilisation que vous pouvez consulter en ligne.

https://apropos.erudit.org/fr/usagers/politique-dutilisation/ 
Thomas Nagel, Égalité et partialité (traduit par Cl. Beauvillard), Paris, Presses Universitaires de France (coll. "Philosophie morale"), 1994, vi-199 pages.

Ce livre de Thomas Nagel a comme ambition de déployer dans le cadre de la philosophie politique, et de la justice sociale. la distinction entre le point de vue personnel et le point de vue impersonnel. dont Le point de vue de nulle part (1986 : trad. fr. 1993) avait analysé avec finesse les tenants et les aboutissants pour la vie morale privée. Les résultats de cette nouvelle entreprise sont, selon moi, assez décevants. Certes Nagel demeure un auteur intelligent et intéressant au contact duquel on peut toujours s'enrichir et dont les convictions morales me semblent, pour la plupart, foncièrement justes, mais là n'est pas la question. Égalité et partialité est un livre qui n'offre à la philosophie politique que bien peu qui soit neuf, tant au niveau des thèses substantielles qu'il défend qu'au niveau des modes de légitimation, dont Nagel fait pourtant un de ses thèmes majeurs. Nous y reviendrons par la suite. Pis, selon moi, la façon particulière dont Nagel propose de comprendre la distinction entre le point de vue personnel et le point de vue impersonnel appliquée à la philosophie politique me semble susceptible 
d'engendrer la confusion plus que de jeter un éclairage nouveau et intéressant sur les problemes traditionnels de la discipline. C'est du moins ce que j'aimerais tenter de montrer dans la suite de ce court compte rendu.

Une des difficultés centrales de la vie morale pour Nagel, on le sait, c'est de réussir à concilier le point de vue impersonnel, universel et impartial, caractéristique de la réflexion éthique, avec le regard toujours entaché de partialité que l'individu porte sur le monde. La force de ses travaux précédents est d'avoir su montrer, de façon très convaincante, combien le point de vue personnel compte du point de vue impersonnel même, et combien il est difficile d'établir et de maintenir entre l'un et l'autre l'équilibre enchevêtré qui s'impose. Il est peu étonnant dès lors qu'il pense retrouver cette difficulté dans le domaine de la philosophie politique et qu'il la situe aux environs du problème de la légitimité politique. Car si un régime n'est légitime que si ceux qui lui sont soumis le reconnaissent, il s'impose alors qu'il lui faudra répondre tant à l'une qu'à l'autre dimension de la vie morale.

Or si cette hypothèse est raisonnable, mème si elle laisse ouverte la question de ce qui relève de la légitimité et de ce qui relève de la simple stabilité d'un régime politique, l'interprétation que Nagel en propose me semble fautive et faire l'économie justement de ce qui constitue la particularité de la philosophie politique, par rapport à la morale individuelle, à savoir : la pluralité des agents. Dès le début de l'ouvrage, dès les pages un et deux. Nagel pose que la difficulté d'accorder les visées personnelles et impersonnelles dans le domaine moral nous est depuis longtemps familière en politique puisqu'il s'agit du problème de concilier le point de vue de la collectivité et celui de l'individu. "Le point de vue impersonnel, nous dit-il, représente les exigences de la collectivité et incarne, aux yeux de chaque individu, leur force de conviction"(p. 2), le point de vue personnel, à l'opposé, nourrit les exigences de l'individu. Il en conclut par um raccourci pressé que les "problèmes les plus ardus de la théorie politique résident dans les conflits surgissant chez l'individu " (p. 2). Au delà du caractère à tout le moins étonnant de cette transmutation des problèmes politiques en des conflits intérieurs à l'individu, métamorphose qui suppose au préalable la réduction de la théorie politique à la seule question de la légitimité, l'identification des points de vues personnels et impersonnels aux exigences de l'individu d'une part et de la communauté de l'autre est fausse et trompeuse.

L'erreur vient de ce que le point de vue impersonnel est un point de vue impartial, objectif, et égalitaire tandis que, il suffit de regarder autour de soi. les exigences que les collectivitès font peser sur leurs membres ne sont pas toutes, loin s'en faut, de cette qualité-là. Nagel, il est vrai, en un sens le reconnait, mais il n'en tire pas selon moi la conclusion qui s'impose, puisqu'il range en conséquence toutes les revendications des divers groupes dans le point de vue de l'individu ! On se demande ce qui peut rester au sein du point de vue de la collectivité. En fait, on pourrait tout aussi aisément, je crois, arguer le contraire de ce que dit Nagel, et avancer que les revendications de l'individu contre la tyrannie du groupe sont le résultat du point de vue impersonnel et que les exigences de la communauté ne représentent que la convergence, toujours fortuite, de visées personnelles. Entre ces deux interprétations, il n'y a pas à choisir. L'une et l'autre sont trop incertaines, trop sujettes à d'innombrables contre-exemples, pour qu'on puisse la plaquer sur les rapports entre l'individu et la communauté. Ce n'est pas que la distinction entre les points de vue personnels et impersonnels soit sans intérèt ni pertinence pour la philosophie politique, mais il est naüf et erroné d'y voir soit le modèle. soit l'image de la division entre l'individu et la collectivité. 
La source de cette erreur transparaît lorsque Nagel en vient à traiter plus précisément de la question de la légitimité politique. Il se toume alors vers Kant afin de trouver une forme d'unanimité qui permette de concilier les deux points de vue et il conclut avec lui que la seule unanimité possible dans une société dêmocratique moderne est une unanimité idéale plutôt que réelle. Cependant, il est difficile de dire quel sens exact Nagel accorde à la notion d'" unanimité idéale ". Par moment il semble penser, comme Kant, qu'il s'agit du consensus de la communauté idéale de tous les êtres rationnels, c'est-à-dire d'une communauté qui ne peut exister qu'en pensée. Par moment il s'agit semble-t-il du consensus idéal d'une communauté réelle d'ètres rationnels. C'est-à-dire, ce dont tous les membres de la communauté conviendraient s'ils agissaient de façon rationnelle. Or si le point de vue de nulle part représente peut-ètre les exigences unanimes de la communauté idéale de tous les étres rationnels, il ne correspond certainement pas aux revendications d'une communauté d'ètres réels, fussent-ils parfaitement rationnels, pour l'excellente raison que le point de vue de nulle part est sans particularité. Les collectivités politiques sont des communautés réelles. C'est parce que Nagel a réduit à une tension interne le conflit politique qu'il peut penser les liens entre l'individu et la collectivité comme l'articulation entre l'idéal (et l'idéel) et le réel.

En conséquence de l'imprécision qui la grève, l'analyse que Nagel propose de la position kantienne reste, je crois, bien en deçà de celle qu'a faite Onora O'Neil (Constructions of Reason: Explorations in Kant's Practical Philosophy. Cambridge University Press, 1988) et il faut avouer que sur presque toutes les questions de justice sociale ses positions sont, à peu de choses près, celles qui sont défendues par d'autres auteurs comme R. Dworkin, J. Rawls, J. Raz ou T. Scanlon. Nagel le reconnait, mais on aurait espéré qu'il offrit des justifications originales ou une compréhension nouvelle de ces partis-pris connus. Nagel, il est vrai, défend un égalitarisme plus fort, mais pas nécessairement plus conséquent (voir à ce sujet le chapitre 12, "L'inégalité "), que celui mis de l'avant par la plupart de ces auteurs. Malheureusement, ici encore, le lecteur en quête de justification reste sur sa faim. Il nous est dit simplement que cet égalitarisme découle de l'impartialité caractéristique du point de vue impersonnel. comme si cela allait de soi.

À sa décharge, il faut dire enfin que Nagel n'est pas toujours très bien servi par sa traductrice. En guise de conclusion, j'invite le lecteur à tenter de comprendre la phrase suivante : "La croyance est raisonnable quand elle est fondée sur une donnée qui ne suffit pas à conclure et sur le jugement que l'on porte. "(p. 173)

Paul Dumouchel

Institute for Research in Humanities Kyoto University 